University of New Orleans

ScholarWorks@UNO

$1-2002$

\title{
Angular range for reflection of p-polarized light at the surface of an absorbing medium with reflectance below that at normal incidence
}

\author{
R. M.A. Azzam \\ University of New Orleans, razzam@uno.edu \\ Ericson E. Ugbo
}

Follow this and additional works at: https://scholarworks.uno.edu/ee_facpubs

Part of the Electrical and Electronics Commons, and the Optics Commons

\section{Recommended Citation}

R. M. A. Azzam and Ericson E. Ugbo, "Angular range for reflection of p-polarized light at the surface of an absorbing medium with reflectance below that at normal incidence," J. Opt. Soc. Am. A 19, 112-115 (2002)

This Article is brought to you for free and open access by the Department of Electrical Engineering at ScholarWorks@UNO. It has been accepted for inclusion in Electrical Engineering Faculty Publications by an authorized administrator of ScholarWorks@UNO. For more information, please contact scholarworks@uno.edu. 


\title{
Angular range for reflection of $p$-polarized light at the surface of an absorbing medium with reflectance below that at normal incidence
}

\author{
R. M. A. Azzam \\ Department of Electrical Engineering, University of New Orleans, New Orleans, Louisiana 70148 \\ Ericson E. Ugbo \\ 8152 Drais Way, Elk Grove, California 95624
}

Received April 30, 2001; accepted June 20, 2001

\begin{abstract}
The range of incidence angle, $0<\varphi<\varphi_{\mathrm{e}}$, over which $p$-polarized light is reflected at interfaces between transparent and absorbing media with reflectance below that at normal incidence is determined. Contours of constant $\varphi_{\mathrm{e}}$ in the complex plane of the relative dielectric constant $\varepsilon$ are presented. A method for determining the real and imaginary parts of the complex refractive index, $\varepsilon^{1 / 2}=n+j k$, which is based on measuring $\varphi_{\mathrm{e}}$ and the pseudo-Brewster angle $\varphi_{\mathrm{pB}}$, is viable in the domain of fractional optical constants, $n, k<1$. (C) 2002 Optical Society of America
\end{abstract}

OCIS codes: $240.0240,260.0260,260.3910,260.5430,290.3030$.

\section{INTRODUCTION}

The reflection of collimated monochromatic $p$-polarized light at the planar interface between a transparent medium of incidence of real dielectric constant $\varepsilon_{1}$ and an absorbing medium of refraction of complex dielectric constant $\varepsilon_{2}$ is governed by the Fresnel reflection coefficient ${ }^{1}$

$$
\begin{aligned}
r_{p}=[ & \left.\varepsilon \cos \varphi-\left(\varepsilon-\sin ^{2} \varphi\right)^{1 / 2}\right] /[\varepsilon \cos \varphi \\
& \left.+\left(\varepsilon-\sin ^{2} \varphi\right)^{1 / 2}\right],
\end{aligned}
$$

where

$$
\varepsilon=\varepsilon_{2} / \varepsilon_{1}
$$

and $\varphi$ is the angle of incidence; see Fig. 1. For a given complex $\varepsilon$, the absolute reflectance $r_{p} r_{p}^{*}$ initially decreases as $\varphi$ increases from 0 , reaches a minimum at the pseudo-Brewster angle $\varphi_{\mathrm{pB}}$, and then increases monotonically from minimum reflectance to 1 as $\varphi$ increases from $\varphi_{\mathrm{pB}}$ to $90^{\circ}$. Explicit solutions for $\varphi_{\mathrm{pB}}$ for a given complex $\varepsilon$ have been derived by several authors. ${ }^{2-4} \mathrm{Az}$ zam and $\mathrm{Ugbo}^{5}$ also determined analytically the contours of constant $\varphi_{\mathrm{pB}}$ in the complex $\varepsilon$ plane.

In this paper we are interested in the angular range $0<\varphi<\varphi_{\mathrm{e}}$ over which the reflectance for $p$-polarized light at oblique incidence is less than that at normal incidence. The upper limit $\varphi_{\mathrm{e}}$, which lies between $\varphi_{\mathrm{pB}}$ and $90^{\circ}$, is determined by equating the oblique and normalincidence reflectances, i.e.,

$$
r_{p}(\varphi) r_{p}^{*}(\varphi)=r_{p}(0) r_{p}^{*}(0) .
$$

For the special case of an interface between two transparent media ( $\varepsilon$ real and $>0$ ), the minimum reflectance is zero, $\varphi_{\mathrm{pB}}$ reverts to the usual Brewster angle $\varphi_{\mathrm{B}}$ $=\arctan \varepsilon^{1 / 2}$, and Eq. (3) has an explicit solution $\varphi$ $=\varphi_{\mathrm{e}}$ given $\mathrm{by}^{6}$

$$
\tan \varphi_{\mathrm{e}}=\left(\varepsilon^{2}+\varepsilon\right)^{1 / 2}
$$

Another interesting conclusion from Ref. 6 is that the difference $\varphi_{\mathrm{e}}-\varphi_{\mathrm{B}}$ reaches a maximum of $13.9852^{\circ}$ when $\varepsilon$ $=3.6135$, and $\varphi_{\mathrm{B}}=62.2528^{\circ}$.

For the general case of an absorbing medium of refraction (complex $\varepsilon$ ), no analytical solution exists for Eqs. (1) and (3), and $\varphi_{\mathrm{e}}$ must be determined numerically. Our approach in this paper is to determine all possible values of $\varphi_{\mathrm{e}}-\varphi_{\mathrm{pB}}$ that are consistent with a given $\varphi_{\mathrm{pB}}$ (Section 2). The maximum difference $\left(\varphi_{\mathrm{e}}-\varphi_{\mathrm{pB}}\right)_{\max }=20.447^{\circ}$ occurs in the limit when $\varepsilon$ is real negative, and $\varphi_{\mathrm{pB}} \approx 44^{\circ}$. We also determine the constant- $\varphi_{\mathrm{e}}$ contours in the complex planes of $\varepsilon$ and $\varepsilon^{1 / 2}(=n+j k$, the relative complex refractive index) in Section 3. In Section 4, we propose a technique for determining $n$ and $k$, which is based on measuring the two angles $\varphi_{\mathrm{pB}}$ and $\varphi_{\mathrm{e}}$.

\section{ANGULAR RANGE $\varphi_{\mathrm{e}}-\varphi_{\mathrm{pB}}$ FOR SPECIFIED PSEUDO-BREWSTER ANGLE $\varphi_{\mathrm{pB}}$}

All possible values of complex $\varepsilon=|\varepsilon| \exp (j \theta)$, that are consistent with a given $\varphi_{\mathrm{pB}}$ are determined by ${ }^{5}$

$$
|\varepsilon|=\iota \cos (\zeta / 3)
$$




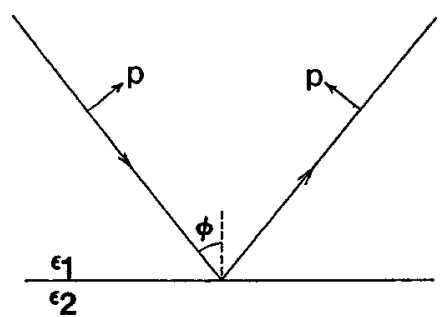

Fig. 1. Reflection of $p$-polarized light at an angle $\varphi$ by the planar interface of two media with dielectric constants $\varepsilon_{1}$ and $\varepsilon_{2}$.

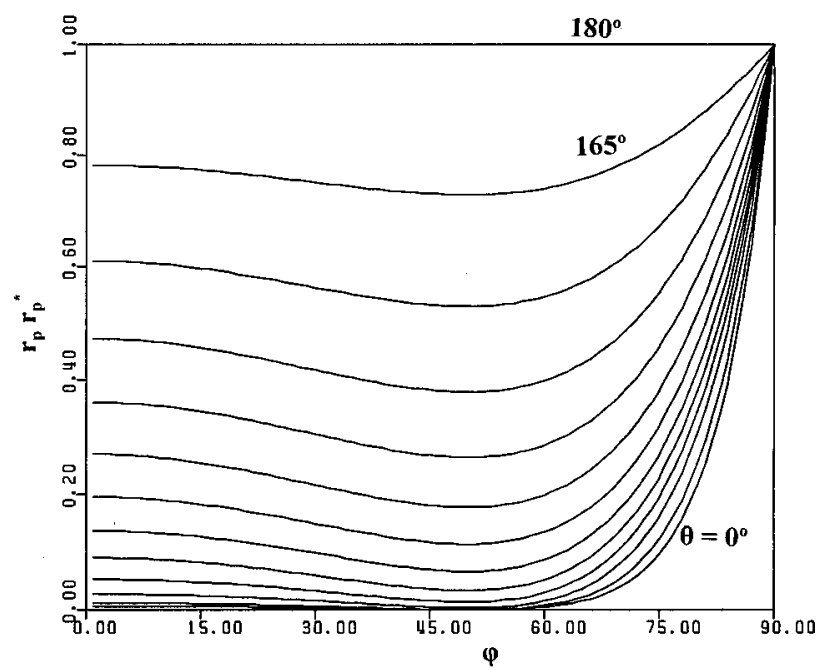

Fig. 2. Family of reflectance-versus-angle $\left(r_{p} r_{p}^{*}\right.$-versus- $\left.\varphi\right)$ curves that share the same pseudo-Brewster angle $\varphi_{\mathrm{pB}}=50^{\circ}$. The associated values of complex $\varepsilon=|\varepsilon| \exp (j \theta)$ are obtained from Eqs. (5)-(7) by allowing $\theta$ to assume values from 0 to $180^{\circ}$ in steps of $15^{\circ}$

where

$$
\begin{gathered}
\iota=2 \tan ^{2} \varphi_{\mathrm{pB}}\left(1-\frac{2}{3} \sin ^{2} \varphi_{\mathrm{pB}}\right)^{1 / 2} \\
\zeta=\arccos \left[-\cos \theta \cos ^{2} \varphi_{\mathrm{pB}}\left(1-\frac{2}{3} \sin ^{2} \varphi_{\mathrm{pB}}\right)^{-3 / 2}\right],
\end{gathered}
$$

by scanning $\theta$ from 0 to $180^{\circ}$. Constant- $\varphi_{\mathrm{pB}}$ contours in the complex $\varepsilon$ plane were presented in Ref. 5 based on Eqs. (5)-(7).

Figure 2 shows a family of reflectance-versus-angle $\left(r_{p} r_{p}^{*}\right.$-versus- $\left.\varphi\right)$ curves for 13 values of complex $\varepsilon$ that share the same pseudo-Brewster angle $\varphi_{\mathrm{pB}}=50^{\circ}$, as obtained by allowing $\theta$ to assume values from 0 to $180^{\circ}$ in steps of $15^{\circ}$. Both the normal-incidence reflectance and the minimum reflectance at $\varphi_{\mathrm{pB}}=50^{\circ}$ increase monotonically with $\theta$. In the limit of $\theta=180^{\circ}$ (i.e., $\varepsilon$ is real negative), the reflectance is total $(=1)$ at all angles.

Figure 3 shows the minimum reflectance, $\left(r_{p} r_{p}^{*}\right)_{\min }$, as a function of $\theta$ for constant values of $\varphi_{\mathrm{pB}}$ from $5^{\circ}$ to $80^{\circ}$ in steps of $5^{\circ}$. For small pseudo-Brewster angles $\left(5^{\circ}\right.$ to $\left.15^{\circ}\right)$, an initial steep rise of the minimum reflectance with $\theta$ is followed by a more gradual increase toward 1 . For $\varphi_{\mathrm{pB}}$ $>30^{\circ}$, the increase of minimum reflectance with $\theta$ appears parabolic and is nearly independent of $\varphi_{\mathrm{pB}}$.

Figure 4 shows $\varphi_{\mathrm{e}}-\varphi_{\mathrm{pB}}$ as a function of $\theta$ for constant $\varphi_{\mathrm{pB}}$ from $5^{\circ}$ to $80^{\circ}$ in steps of $5^{\circ}$. The maximum difference $\left(\varphi_{\mathrm{e}}-\varphi_{\mathrm{pB}}\right)_{\max }=20.447^{\circ}$ occurs when $\theta=180^{\circ}$ and $\varphi_{\mathrm{pB}} \approx 44^{\circ}$. For large values of $\varphi_{\mathrm{pB}},\left(\varphi_{\mathrm{e}}-\varphi_{\mathrm{pB}}\right)$ is nearly constant (e.g., at $\varphi_{\mathrm{pB}}=80^{\circ}, \varphi_{\mathrm{e}}-\varphi_{\mathrm{pB}}$ increases from $8.270^{\circ}$ to $8.351^{\circ}$ as $\theta$ increases from 0 to $180^{\circ}$ ).

\section{CONSTANT- $\varphi_{\mathrm{e}}$ CONTOURS IN THE COMPLEX PLANES OF $\varepsilon$ AND $\varepsilon^{1 / 2}$}

Over the range of incidence angles $0<\varphi<\varphi_{\mathrm{e}}$ the $p$ reflectance at oblique incidence is less than that at normal incidence. It is of interest to consider the constant- $\varphi_{\mathrm{e}}$ contours in the complex $\varepsilon$ plane. Figure 5 shows a family of such contours for $\varphi_{\mathrm{e}}$ from 45 to $80^{\circ}$ in steps of $5^{\circ}$ and $\varphi_{\mathrm{e}}$ from 80 to $85^{\circ}$ in steps of $1^{\circ}$. These results are obtained by solving Eqs. (1) and (3) numerically. The curves resemble a family of semicircles centered at the origin. (However, each contour is not a semicircle.) Figure 6 shows the corresponding family of contours in the complex-refractive-index plane, $\varepsilon^{1 / 2}=n+j k$. The de-

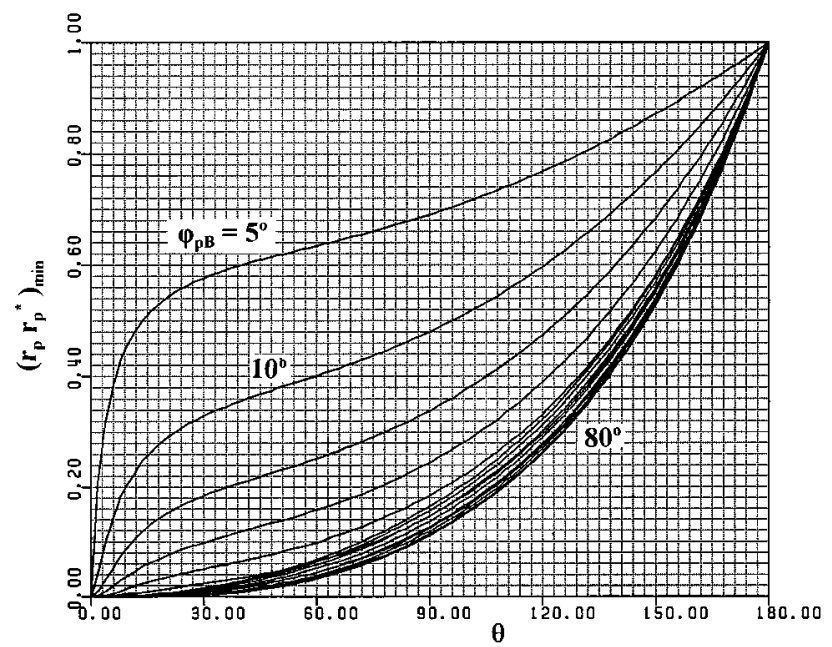

Fig. 3. Minimum reflectance at the pseudo-Brewster angle $\varphi_{\mathrm{pB}},\left(r_{p} r_{p}^{*}\right)_{\min }$, as a function of $\theta$ for constant values of $\varphi_{\mathrm{pB}}$ from 5 to $80^{\circ}$ in steps of $5^{\circ}$.

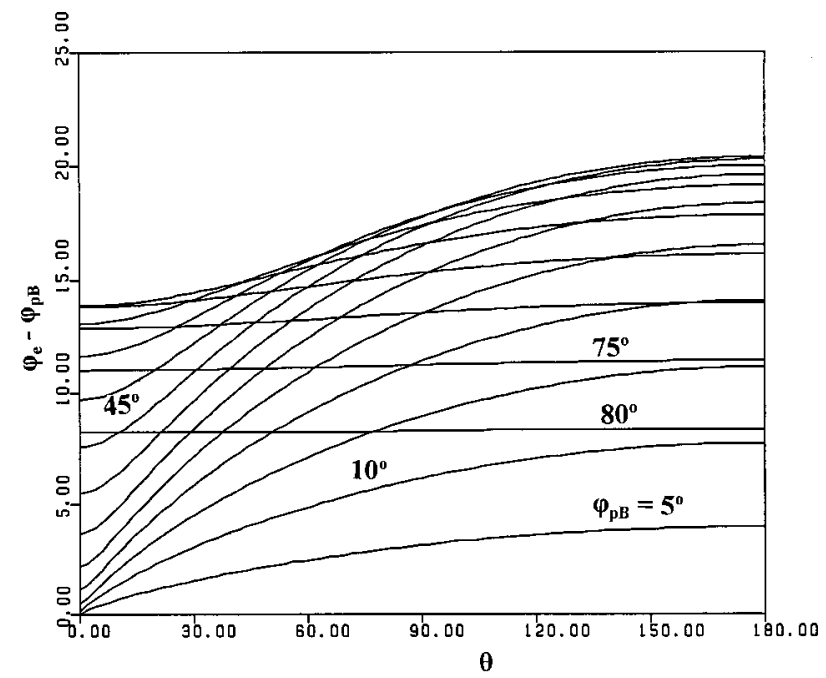

Fig. 4. Angle difference $\varphi_{\mathrm{e}}-\varphi_{\mathrm{pB}}$ as a function of $\theta$, for constant values of $\varphi_{\mathrm{pB}}$ from 5 to $80^{\circ}$ in steps of $5^{\circ} . \varphi_{\mathrm{e}}$ defines the upper limit of the range of incidence angle for which the $p$ reflectance at oblique incidence is less than that at normal incidence. 


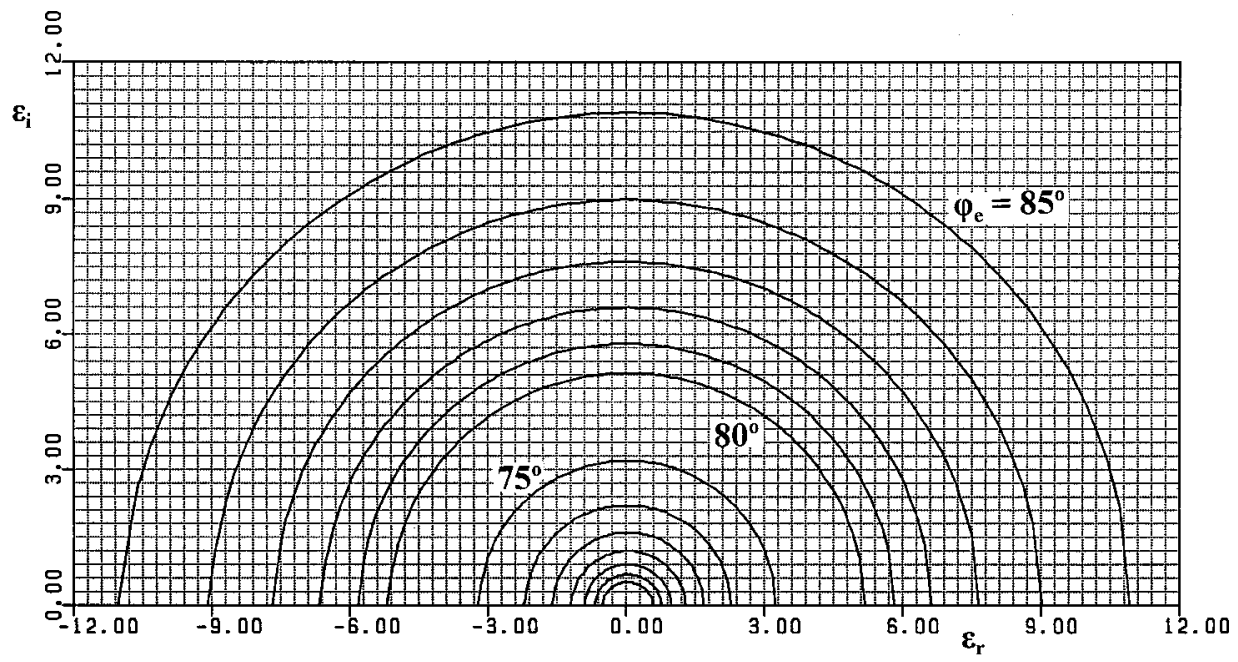

Fig. 5. Family of contours of constant $\varphi_{\mathrm{e}}=45$ to $80^{\circ}$ in steps of $5^{\circ}$, and $\varphi_{\mathrm{e}}=80$ to $85^{\circ}$ in steps of $1^{\circ}$. $\varphi_{\mathrm{e}}$ defines the upper limit of the range of incidence angle for which the $p$ reflectance at oblique incidence is less than that at normal incidence.

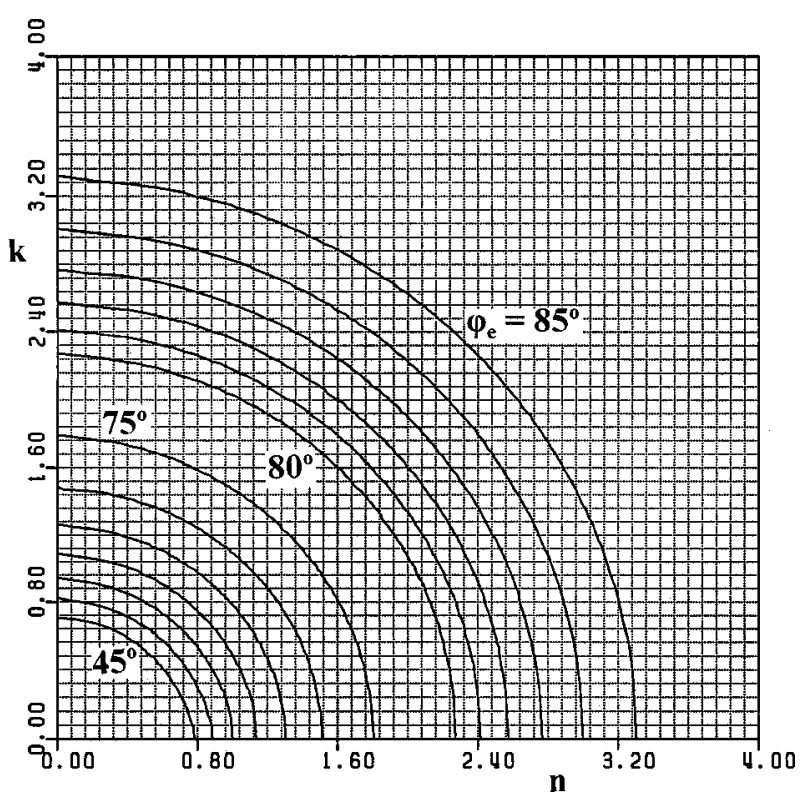

Fig. 6. Family of constant- $\varphi_{\mathrm{e}}$ contours in the nk complex refractive index plane for the same values of $\varphi_{\mathrm{e}}$ as in Fig. 5.

viation of each contour from a quadrant of a circle is more apparent at lower angles (e.g., at $\varphi_{\mathrm{e}}=45^{\circ}$ ).

\section{TECHNIQUE FOR DETERMINING $n$ AND $k$ FROM THE MEASURED ANGLES $\varphi_{\mathrm{pB}}$ AND $\varphi_{\mathbf{e}}$}

Azzam described an analytical technique for determining the optical constants $n$ and $k$ of an absorbing medium from two pseudo-Brewster angles measured in two transparent incidence media. ${ }^{7}$ It is of interest to consider whether $n$ and $k$ can be determined from the two angles $\varphi_{\mathrm{pB}}$ and $\varphi_{\mathrm{e}}$ measured in the same medium of incidence. In general, angular measurements are attractive, because no absolute reflectance measurements are required. (For

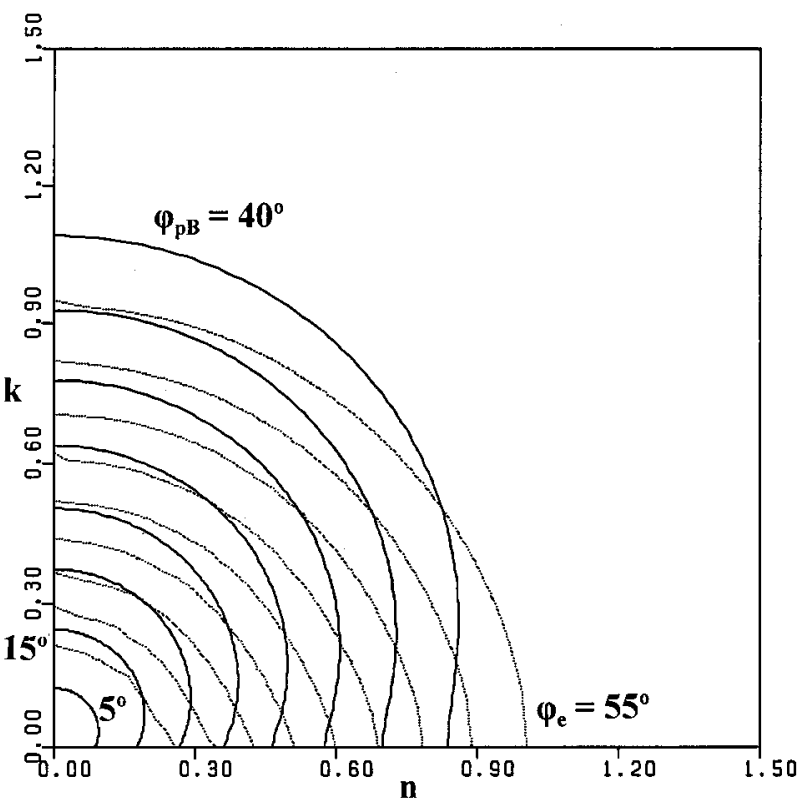

Fig. 7. Families of constant $-\varphi_{\mathrm{pB}}$ and constant- $\varphi_{\mathrm{e}}$ contours in the $n-k$ plane in the domain of fractional optical constants $(n, k$ $<1$ ).

a review of numerous reflectance-based techniques, the reader may consult papers by Humphreys-Owen ${ }^{2}$ and Hunter. ${ }^{8}$ )

Figure 7 shows two superimposed families of constant$\varphi_{\mathrm{pB}}$ and constant- $\varphi_{\mathrm{e}}$ contours in the $n-k$ plane in the domain of fractional optical constants. This domain is important in that it relates to attenuated total internal reflection when light is incident from a dense medium. The contours are shown for $\varphi_{\mathrm{pB}}=5$ to $40^{\circ}$ in steps of $5^{\circ}$ and for $\varphi_{\mathrm{e}}=15$ to $55^{\circ}$ in steps of $5^{\circ}$. The angles of intersection of curves of one family with curves of the other provide a measure of the precision with which $n$ and $k$ can be determined. It is apparent from Fig. 7 that $n$ and $k$ can be reasonably well determined when $k<n$.

Figure 8 is similar to Fig. 7, except that values of $n, k$ $>1$ are now considered. In Fig. 8 the families of 


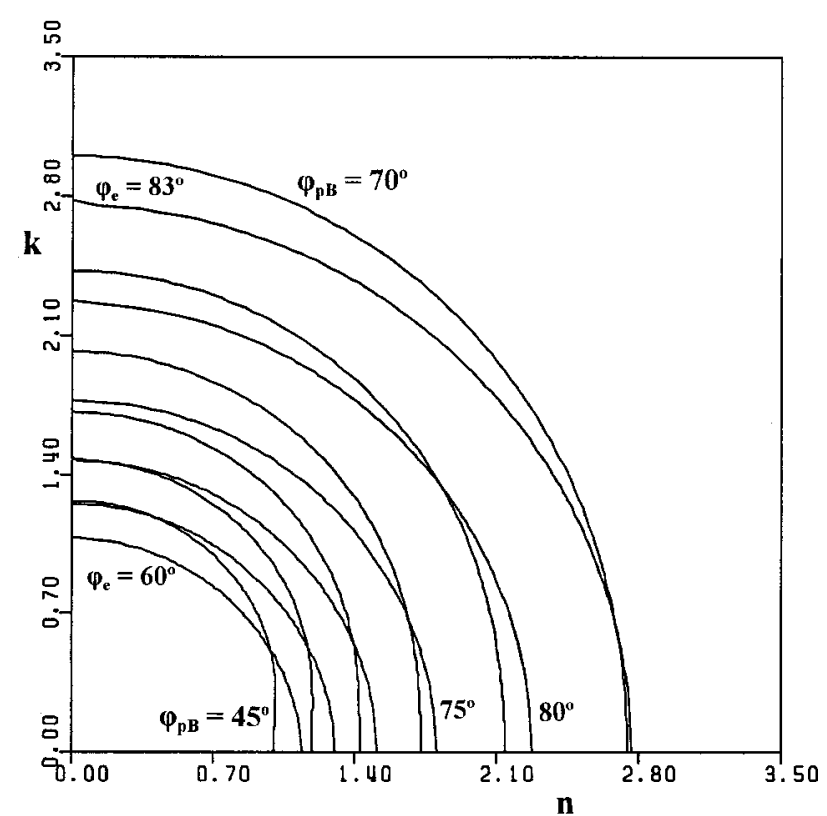

Fig. 8. Families of constant- $\varphi_{\mathrm{pB}}$ and constant- $\varphi_{\mathrm{e}}$ contours in the $n-k$ plane for $n, k>1$.

constant $-\varphi_{\mathrm{pB}}$ and constant $-\varphi_{\mathrm{e}}$ contours are generated for $\varphi_{\mathrm{pB}}=45$ to $70^{\circ}$ in steps of $5^{\circ}$, and for $\varphi_{\mathrm{e}}=60$ to $80^{\circ}$ in steps of $5^{\circ}$, and $\varphi_{\mathrm{e}}=83^{\circ}$. Because of the smaller intersection angles, the present two-angle method would not provide an accurate method of determining $n$ and $k$

\section{SUMMARY}

We have determined the range of incidence angles, 0 $<\varphi<\varphi_{\mathrm{e}}$, over which the reflectance of $p$-polarized light at oblique incidence is less than that at normal incidence, for any transparent medium/absorbing medium interface. Constant $-\varphi_{\mathrm{e}}$ contours in the complex planes of the dielectric constant $\varepsilon$ and refractive index $\varepsilon^{1 / 2}=n+j k$ are obtained. Finally, it is shown that fractional optical constants $n$ and $k$ can be determined if the pseudo-Brewster angle and the angle $\varphi_{\mathrm{e}}$ [which satisfies Eq. (3)] are measured.

\section{REFERENCES}

1. See, for example, R. M. A. Azzam and N. M. Bashara, Ellipsometry and Polarized Light (North-Holland, Amsterdam, 1987), Chap. 4.

2. S. P. F. Humphreys-Owen, "Comparison of reflection methods for measuring optical constants without polarimetric analysis, and proposal for new methods based on the Brewster angle," Proc. Phys. Soc. London 77, 949-957 (1961).

3. R. M. A. Azzam, "Maximum minimum reflectance of parallel-polarized light at interfaces between transparent and absorbing media,” J. Opt. Soc. Am. 73, 959-962 (1983).

4. S. Y. Kim and K. Vedam, "Analytic solution of the pseudoBrewster angle," J. Opt. Soc. Am. A 3, 1772-1773 (1986).

5. R. M. A. Azzam and E. E. Ugbo, "Contours of constant pseudo-Brewster angle in the complex $\varepsilon$ plane and an analytical method for the determination of optical constants," Appl. Opt. 28, 5222-5228 (1989).

6. R. M. A. Azzam, "Equalization of reflectance of parallelpolarized electromagnetic waves at normal and oblique incidence of interfaces between transparent media and its use for measurement of the dielectric constant," Appl. Phys. 20, 193-195 (1979).

7. R. M. A. Azzam, "Analytical determination of the complex dielectric function of an absorbing medium from two angles of incidence of minimum parallel reflectance," J. Opt. Soc. Am. A 6, 1213-1216 (1989).

8. W. R. Hunter, "Measurement of optical constants in the vacuum ultraviolet spectral region," in Handbook of Optical Constants of Solids, E. Palik, ed. (Academic, New York, 1985). 